\title{
Pisa syndrome associated with mirtazapine: a case report
}

\author{
Yuji Yamada ${ }^{1}$, Harumasa Takano ${ }^{1,2^{*}}$ (D, Maki Yamada ${ }^{1}$, Naoko Satake ${ }^{1}$, Naotsugu Hirabayashi ${ }^{1}$, Mitsutoshi Okazaki ${ }^{1}$ \\ and Kazuyuki Nakagome ${ }^{3}$
}

\begin{abstract}
Background: Mirtazapine is a noradrenergic and specific serotonergic antidepressant; its pharmacological profile indicates a low risk for dopaminergic adverse effects. To date, there has been only a single case report of Pisa syndrome associated with mirtazapine.
\end{abstract}

Case presentation: The authors report a case involving a 79-year-old woman with bipolar disorder, in whom Pisa syndrome occurred after introduction of mirtazapine, and completely disappeared 3 days after suspension of the drug.

Conclusions: Aspects of this particular case suggest that Pisa syndrome is a possible side effect of Mirtazapine.

Keywords: Mirtazapine, Side-effects, Dystonia, Pisa syndrome, Bipolar disorder

\section{Background}

Mirtazapine (MTZ) is a noradrenergic and specific serotonergic antidepressant, which is also classified as a tetracyclic antidepressant [1]. Because MTZ does not strongly block the dopamine D1/D2 receptor, there are few reports describing parkinsonism caused by the drug. In the three clinical trials undertaken in Japan, the incidence of dyskinesia was $0-1.0 \%$ but there was no report in terms of parkinsonism or dystonia [2]. In the English literature, there are only two case reports describing dystonia with MTZ use, with one involving Pisa syndrome [3, 4], both of which are cases of major depression. Pisa syndrome is a type of dystonia originally described by Ekbom et al. (1972) [5], which produces abnormally sustained posture with a lateral inclination of the trunk and a degree of backward axial rotation. Drug-induced Pisa syndrome is commonly observed in patients undergoing long-term antipsychotic treatment. However, it has also been reported-albeit less frequently-in patients who receive other medications such as tricyclic antidepressants, selective

\footnotetext{
* Correspondence: hrtakano@ncnp.go.jp

${ }^{1}$ Department of Psychiatry, National Center Hospital, National Center of Neurology and Psychiatry, 4-1-1 Ogawahigashi-cho, Kodaira, Tokyo 187-8551, Japan

${ }^{2}$ Department of Clinical Neuroimaging, Integrative Brain Imaging Center, National Center of Neurology and Psychiatry, Tokyo, Japan

Full list of author information is available at the end of the article
}

serotonin reuptake inhibitors, cholinesterase inhibitors, antiemetic drugs, lithium carbonate, benzodiazepines, and tiapride [6]. The present report describes a case of Pisa syndrome that manifested after administration of MTZ to a patient previously diagnosed with bipolar disorder.

\section{Case report}

The patient was a 79-year-old Japanese woman, who provided informed consent for publication of anonymized case details. She had a history of high blood pressure, which had been treated with $80 \mathrm{mg} /$ day valsar$\tan$ and $5 \mathrm{mg} /$ day amlodipine since the age of 55 . No family history of epilepsy or movement disorders was noted. She was diagnosed with bipolar disorder when she was 64 years of age, and treatment was started with $200 \mathrm{mg} /$ day lithium carbonate and $400 \mathrm{mg} /$ day sodium valproate. At least four depressed and five manic phases appeared between ages 64 and 79 years. At 79 years of age, she was admitted to the authors' hospital with serious depression. Three months before the admission at 79 years of age for severe depression, olanzapine was used to treat mania for 6 weeks with a maximum dose of $12.5 \mathrm{mg} /$ day. Two weeks before the admission, $12.5 \mathrm{mg} /$ day quetiapine was added to treat depression. There were no adverse effects including extrapyramidal symptoms during her previous treatment. Once $15 \mathrm{mg} /$ day MTZ was administered, quetiapine was tapered off

(c) The Author(s). 2018 Open Access This article is distributed under the terms of the Creative Commons Attribution 4.0 International License (http://creativecommons.org/licenses/by/4.0/), which permits unrestricted use, distribution, and 
within 7 days. Three days after administration of $15 \mathrm{mg} /$ day MTZ, she exhibited symptoms of parkinsonism including short-step gait, rigidity, and tremor. Moreover, 12 days after initiation of MTZ administration, when the dose level had been raised to $22.5 \mathrm{mg} /$ day for 5 days, she presented with an abnormal maintained posture of the trunk, which had a lateral deviation to the right side. No other localizing signs were found, and no changes had been made to her usual medication after the introduction of MTZ. Blood and biochemical screening, and brain computed tomography were normal. Although her cognitive function was preserved (Mini-Mental State Examination score 22/30), the possibility of developing Parkinson's disease or Lewy body disease was considered. Brain perfusion single-photon emission computed tomography with ${ }^{99 \mathrm{~m}}$ Tc-ethyl cistainate dimer revealed mild decreases in cerebral blood flow in both sides of the frontal lobe. Myocardial metaiodobenzylguanidine (MIBG) scintigraphy revealed no dysfunction in the sympathetic nerves. Dopamine transporter imaging with ${ }^{123}$ I ioflupane did not reveal nigrostriatal degeneration. From the results of these imaging tests, the possibility of idiopathic Parkinson's disease or Lewy body disease was ruled out. The patient was diagnosed with Pisa syndrome and the MTZ was quickly tapered off; 3 days later, dystonia completely disappeared.

\section{Discussion}

In this case, 12 days after initiation of MTZ administration, when the dose level reached $22.5 \mathrm{mg} /$ day for 5 days, the patient presented with Pisa syndrome; 3 days after suspension of the drug, the syndrome completely disappeared. Furthermore, the results of imaging tests ruled out the possibility of organic disease(s). Accordingly, Pisa syndrome was suspected to have been associated with MTZ.

This is the second report of Pisa syndrome associated with MTZ. Moreover, this is the first case report of Pisa syndrome in which the possibility of idiopathic Parkinson's disease and Lewy body disease was carefully excluded using MIBG scintigraphy and dopamine transporter imaging with ${ }^{123}$ I ioflupane.

The mechanism through which MTZ produces Pisa syndrome remains unclear. The first possible explanation comes from the fact that MTZ has the largest capacity of all antidepressants to bind to the dopamine D1/D2 receptor $[1,6,7]$. Specifically, the dopamine D1 receptor's half maximal (50\%) inhibitory concentration (IC50) of MTZ is $1600 \mathrm{nM}$, and the dopamine D2 receptor's IC50 is 2500 nM. However, antipsychotic drugs, such as quetiapine, which had a much larger binding capacity to the dopamine D1/D2 receptor than MTZ, did not cause Pisa syndrome in this patient. The second possible explanation is that noradrenergic-dopaminergic imbalance produced Pisa syndrome $[6,8]$. More precisely, it is known that noradrenaline metabolism is impaired in dystonia, so markedly increased noradrenaline level compared to dopamine level in substantia nigra and red nucleus is found in patients with dystonia $[9,10]$. Thus, much higher concentrations of noradrenaline in those areas than those of dopamine associated with MTZ may have produced Pisa syndrome. However, in contrast to this view, it is suggested that MTZ induces the enhancement of the output of dopamine mediated via blockade of $\alpha 2$-adrenergic receptors and facilitation of post-synaptic 5-HT1A function [11]. The last potential explanation is the enhancement of the action of MTZ possibly induced by pharmacokinetic drug-drug interactions. As biotransformation of MTZ is mainly mediated by cytochrome P450(CYP)2D6 and CYP3A4 isoenzymes, inhibitors of these isoenzymes may cause modest increase in MTZ plasma concentrations. However, the adjunctive drugs administered to the patient, such as quetiapine, lithium carbonate, sodium valproate, valsartan and amlodipine, do not affect these isoenzymes [12]. Therefore, drug-drug interactions have a low possibility to be a cause of Pisa syndrome in this patient. In addition, one case report described tardive dystonia induced by the calcium-channel blocker amlodipine [13] Moreover, a review reported that $5 \%$ of drug-induced parkinsonism was caused by calcium-channel blocker [14]. Thus, amlodipine is a potentially suspect drug to induce Pisa syndrome. The review reported that $69 \%$ of drug-induced parkinsonism cases developed during the first 3 months after introduction of calcium-channel blockers, with an additional 20\% within 12 months [14]. Research has suggested that Pisa syndrome should have occurred within 1 year after drug introduction, if amlodipine is the causative agent. However, the present patient had been using amlodipine for more than 20 years. Therefore, there is little possibility of amlodipine-induced Pisa syndrome in this case.

\section{Conclusion}

We report the second case of Pisa syndrome that occurred after the introduction of MTZ, and completely disappeared 3 days after suspension of the drug. Aspects of this particular case suggest that Pisa syndrome is a possible side effect of MTZ.

\section{Acknowledgements}

We would like to thank all the participants who took part in the treatment and the staffs at the National Center Hospital, National Center of Neurology and Psychiatry in Tokyo, Japan.

Funding

None declared.

Availability of data and materials

The dataset supporting the conclusions of this article is available with the corresponding author and will be made available on reasonable request. 


\section{Authors' contributions}

YY was a major contributor in writing the manuscript. HT and $\mathrm{KN}$ assisted in the preparation of the manuscript and revised it critically for important intellectual content. YY, HT, MY, and NS acquired and analyzed data of the case. $\mathrm{HT}, \mathrm{NH}, \mathrm{MO}$, and $\mathrm{KN}$ analyzed and interpreted it. All authors critically reviewed and approved the final manuscript.

\section{Ethics approval and consent to participate}

Not applicable.

\section{Consent for publication}

We obtained written informed consent for publication of anonymized case details from the patient.

\section{Competing interests}

HT has received grants from Biogen Japan, Eli Lilly Japan, Nippon Boehringer Ingelheim, Sumitomo Dainippon Pharma, Meiji Seika Pharma, Nihon MediPhysics, Janssen Pharmaceutical, Tanabe Mitsubishi Pharma, Otsuka Pharmaceutical, and Mochida Pharmaceutical, and speaker's honoraria from Biogen Japan. NS received research funding from Meiji Seika Pharma and Sumitomo Dainippon Pharma. MO received research funding from Eisai,Janssen Pharmaceutical,Meiji Seika Pharma,MSD,Novartis Pharma,Otsuka Pharmaceutical,Quintiles Pharma,UCB Japan,Sumitomo Dainippon Pharma. KN has received lecture fees from Kyowa Hakko Kirin, Otsuka Pharmaceutical, Sumitomo Dainippon Pharma, Eli Lilly Japan, Shionogi, Meiji Seika Pharma, Pfizer Japan, MSD, GlaxoSmithKline, Janssen Pharmaceutical, Mochida Pharmaceutical, and Yoshitomiyakuhin, and participated in an advisory board for Otsuka Pharmaceutical, Nippon Boehringer Ingelheim, Takeda Pharmaceutical, Toyama Chemical, Sumitomo Dainippon Pharma, Meiji Seika Pharma, Pfizer Japan, Janssen Pharmaceutical, and Mochida Pharmaceutical, and received research funding from Mitsubishi Tanabe Pharma, Asahi Kasei Pharma, Astellas Pharma, Mochida Pharmaceutical, Meiji Seika Pharma, Otsuka Pharmaceutical, Nippon Boehringer Ingelheim, and Sumitomo Dainippon Pharma.

\section{Publisher's Note}

Springer Nature remains neutral with regard to jurisdictional claims in published maps and institutional affiliations.

\section{Author details}

${ }^{1}$ Department of Psychiatry, National Center Hospital, National Center of Neurology and Psychiatry, 4-1-1 Ogawahigashi-cho, Kodaira, Tokyo 187-8551, Japan. ${ }^{2}$ Department of Clinical Neuroimaging, Integrative Brain Imaging Center, National Center of Neurology and Psychiatry, Tokyo, Japan. ${ }^{3}$ National Institute of Mental Health, National Center of Neurology and Psychiatry, Tokyo, Japan.

Received: 4 July 2018 Accepted: 21 November 2018

Published online: 06 December 2018

\section{References}

1. de Boer T. The pharmacologic profile of mirtazapine. J Clin Psychiatry. 1996; 57(Suppl 4):19-25.

2. Pharmaceuticals and Medical Device Agency. Common Technical Document of mirtazapine (2.7.4). 2009; http://www.pmda.go.jp/drugs/2009/ P200900035/index.html. Accessed 24 May 2018.

3. Mendez Guerrero A, Llamas S, Murcia FJ, Ruiz J. Acute Pisa syndrome after administration of a single dose of mirtazapine. Clin Neuropharmacol. 2013; 36:133-4. https://doi.org/10.1097/WNF.0b013e318297593d.

4. Lu R, Hurley AD, Gourley M. Dystonia induced by mirtazapine. J Clin Psychiatry. 2002;63:452-3.

5. Ekbom K, Lindholm H, Ljungberg L. New dystonic syndrome associated with butyrophenone therapy. Z Neurol. 1972;202:94-103.

6. Suzuki T, Matsuzaka H. Drug-induced Pisa syndrome (pleurothotonus): epidemiology and management. CNS Drugs. 2002;16:165-74.

7. Hyttel J. Pharmacological characterization of selective serotonin reuptake inhibitors (SSRIs). Int Clin Psychopharmacol. 1994;9(Suppl 1):19-26.

8. Remington GJ. The Pisa syndrome: possible role for serotonin and noradrenaline. J Clin Psychopharmacol. 1988;8:228-9.

9. Hornykiewicz O, Kish SJ, Becker LE, Farley I, Shannak K. Brain neurotransmitters in dystonia musculorum deformans. N Engl J Med. 1986; 315:347-53.
10. Jankovic J. Brain neurotransmitters in dystonia. N Engl J Med. 1987;316:278-9.

11. Nakayama K, Sakurai T, Katsu H. Mirtazapine increases dopamine release in prefrontal cortex by 5-HT1A receptor activation. Brain Res Bull. 2004;63(3): 237-41.

12. Timmer CJ, Sitsen JM, Delbressine LP. Clinical pharmacokinetics of mirtazapine. Clin Pharmacokinet. 2000;38(6):461-74.

13. Dressler D. Tardive dystonic syndrome induced by the calcium-channel blocker amlodipine. J Neural Transm. 2014;121(4):367-9.

14. Bondon-Guitton E, Perez-Lloret S, Bagheri H, Brefel C, Rascol O, Montastruc IL. Drug-induced parkinsonism: a review of 17 years' experience in a regional pharmacovigilance center in France. Mov Disord. 2011;26(12):2226-31.

\section{Ready to submit your research? Choose BMC and benefit from:}

- fast, convenient online submission

- thorough peer review by experienced researchers in your field

- rapid publication on acceptance

- support for research data, including large and complex data types

- gold Open Access which fosters wider collaboration and increased citations

- maximum visibility for your research: over $100 \mathrm{M}$ website views per year

At BMC, research is always in progress.

Learn more biomedcentral.com/submissions 